OPEN ACCESS

Edited by:

Mark Smeltzer,

University of Arkansas for Medical

Sciences, United States

Reviewed by:

Ajmal Khan,

University of Nizwa, Oman

Alexia Hapeshi,

University of Warwick,

United Kingdom

*Correspondence:

Ewa Klara Stuermer

e.stuermer@uke.de

Specialty section:

This article was submitted to

Antimicrobials, Resistance

and Chemotherapy

a section of the journal

Frontiers in Microbiology

Received: 04 February 2021 Accepted: 19 April 2021

Published: 14 May 2021

Citation:

Stuermer EK, Plattfaut I,

Dietrich M, Brill F, Kampe A, Wiencke V, Ulatowski A, Geffken M, Rembe J-D, Naumova EA, Debus SE and Smeets $R$ (2021) In vitro Activity of Antimicrobial Wound Dressings on

$P$. aeruginosa Wound Biofilm.

Front. Microbiol. 12:664030.

doi: 10.3389/fmicb.2021.664030

\section{In vitro Activity of Antimicrobial Wound Dressings on $P$. aeruginosa Wound Biofilm}

\author{
Ewa Klara Stuermer ${ }^{1 *}$, Isabell Plattfaut ${ }^{2}$, Michael Dietrich ${ }^{2}$, Florian Brill ${ }^{3}$, \\ Andreas Kampe ${ }^{3}$, Vanessa Wiencke ${ }^{3}$, Anna Ulatowski ${ }^{3}$, Maria Geffken ${ }^{4}$, \\ Julian-Dario Rembe ${ }^{5}$, Ella Alexandrovna Naumova ${ }^{6}$, Sebastian Eike Debus ${ }^{1}$ and \\ Ralf Smeets 7,8
}

\begin{abstract}
' Department of Vascular Medicine, University Heart Center, University Medical Center Hamburg-Eppendorf (UKE), Hamburg, Germany, ${ }^{2}$ Institute of Virology and Microbiology, Faculty of Health, Centre for Biomedical Education and Research (ZBAF), Witten/Herdecke University, Witten, Germany, ${ }^{3}$ Dr. Brill + Partner GmbH, Institute for Hygiene and Microbiology, Hamburg, Germany, ${ }^{4}$ Institute for Transfusion Medicine, University Medical Center Hamburg-Eppendorf, Hamburg, Germany, ${ }^{5}$ Department of Vascular and Endovascular Surgery, Heinrich-Heine University Düsseldorf, Düsseldorf, Germany, ${ }^{6}$ Department of Biological and Material Sciences in Dentistry. Faculty of Health, School of Dentistry, Witten/Herdecke University, Witten, Germany, ${ }^{7}$ Department of Oral and Maxillofacial Surgery, University Medical Center Hamburg-Eppendorf, Hamburg, Germany, ${ }^{8}$ Department of Oral and Maxillofacial Surgery, Division of Regenerative Orofacial Medicine, University Medical Center Hamburg Eppendorf, Hamburg, Germany
\end{abstract}

The treatment of acute and chronic infected wounds with residing biofilm still poses a major challenge in medical care. Interactions of antimicrobial dressings with bacterial load, biofilm matrix and the overall protein-rich wound microenvironment remain insufficiently studied. This analysis aimed to extend the investigation on the efficacy of a variety of antimicrobial dressings using an in vitro biofilm model (IhBIOM) mimicking the specific biofilm-environment in human wounds. Four wound dressings containing polyhexanide (PHMB), octendine di-hydrochloride (OCT), cadexomer-iodine (C-IOD) or ionic silver (AG) were compared regarding their antimicrobial efficacy. Quantitative analysis was performed using a quantitative suspension method, separately assessing remaining microbial counts within the solid biofilm as well as the dressing eluate (representing the absorbed wound exudate). Dressing performance was tested against $P$. aeruginosa biofilms over the course of 6 days. Scanning electron microscopy (SEM) was used to obtain qualitative visualization on changes in biofilm structure. C-IOD demonstrated superior bacterial reduction. In comparison it was the only dressing achieving a significant reduction of more than $7 \log _{10}$ steps within 3 days. Neither the OCT- nor the AG-containing dressing exerted a distinct and sustained antimicrobial effect. PHMB achieved a non-significant microbicidal effect (1.71 $\pm 0.31 \log _{10}$ steps) at day 1 . Over the remaining course (6 days) it demonstrated a significant microbistatic effect compared to OCT, AG and the control. Quantitative results in the dressing eluate correlate with those of the solid biofilm model. Overall, AG- and OCT-containing dressings did not achieve the expected anti-biofilm efficacy, while C-IOD performed best. Chemical interaction with the biofilms extrapolymeric substance (EPS), visualized in the SEM, and dressing configuration (agent concentration and release pattern) are 
suspected to be responsible. The unexpected low and diverse results of the tested antimicrobial dressings indicate a necessity to rethink non-debridement anti-biofilm therapy. Focussing on the combination of biofilm-disruptive (for EPS structure) and antimicrobial (for residing microorganisms) features, as with C-IOD, using dehydration and iodine, appears reasonably complementary and an optimal solution, as suggested by the here presented in vitro data.

Keywords: wound biofilm, wound dressing, antimicrobials, wound infection, PHMB, silver, octenidine dihydrochloride, iodine

\section{INTRODUCTION}

Infected wounds, especially chronic wounds populated with biofilm, are one of the greatest challenges in modern wound care (Bowler et al., 2001; James et al., 2008; Demidova-Rice et al., 2012; Magill et al., 2014). Antimicrobial agents often fail in effective biofilm eradication (Percival et al., 2019b; Besser et al., 2020). This results from the symbiotic, multi-species society formed in biofilms by microorganisms, encasing themselves in a protective extrapolymeric substance (EPS), which acts as a shield against biochemical penetration by antimicrobial agents. Lateral resistance gene transfer between species and sub-species, dorment persister cells in the depth of biofilm and tissue as well as an effective biochemical diffusion barrier for active agents are further aspects contributing to the high resilience of microbial biofilms (Percival et al., 2015; Kirketerp-Møller et al., 2020). Even if an antimicrobial substance is capable of effectively eradicating a bacterial strain in its planctonic state, a deep enough penetration to achieve the same reductive efficacy, cannot be ensured in a complex biofilm scenario (Rembe et al., 2020).

Dressings and solutions containing silver, PHMB, octenidine or iodine have demonstrated favorable antimicrobial activity in several in vitro and clinical studies (Koburger et al., 2010; Storm-Versloot et al., 2010; Sibbald et al., 2011; Daeschlein, 2013; To et al., 2016; Dissemond et al., 2017). Anti-biofilm effects are often postulated alongside or were investigated in simple in vitro models, but are lacking validation in more complex test settings or clinical studies (O'Meara et al., 2000, 2013). To reduce the individual patient's burden caused by chronic and infected wounds, prevention and treatment of pathogenic biofilm formation in wounds is a serious and relevant challenge in everyday clinical practice (Guest et al., 2017; Guest et al., 2018). Modern antimicrobial wound dressings represent promising therapeutic options in aiding to master this challenge (Daeschlein, 2013).

Mostly, in vitro evaluation of anti-biofilm activity is performed in models and settings less suited to sufficiently mimic a wound biofilm and the interactions with the human wound microenvironment. Such models and settings include simple single-species-biofilms, lower protein challenge than in clinical wounds, non-human matrix material (e.g., plastic or stainless steel surface) and one-dimensional biofilm structures (Brackman and Coenye, 2016; Shukla et al., 2020). In addition, most studies are not performed with dressings, but with antimicrobial solutions to prove the efficacy of a compound.
However, solutions exhibit an entirely different biochemical and biophysical pattern of distribution, penetration and concentration in contact with a wounds micro-environment and biofilm residing in this environment, compared to wound dressings.

Thus, it is currently unclear whether the anti-biofilm activity of antimicrobial agents embedded in wound dressings is comparable to its antimicrobial liquid counterpart in the wound microenvironment. The presented work addresses this question in vitro using a modified human plasma-based biofilm model (lhBIOM) developed and described earlier by the authors (Besser et al., 2020; Rembe et al., 2020; Stuermer et al., 2021). The model mimics the condition of a wound biofilm by consisting of a bacteria-incorporating, matured, three-dimensional (3D) gel-like biofilm structure surrounded by bacteria-rich human plasma, which is similar to the composition of wound exudate in terms of protein content, carbohydrates, enzymes and bacterial breakdown products (Demidova-Rice et al., 2012; Stuermer et al., 2021).

\section{MATERIALS AND METHODS}

\section{Test Organism and Nutrient Solutions}

Pseudomonas aeruginosa (DSM-939) was cultivated on casein/soy peptone agar plates (CSA) following standard procedures (DIN EN 13727). The first subculture was used for testing. The bacterial suspension was adjusted to a 0.5 McFarland standard (approx. $1.5 \times 10^{8} \mathrm{cfu} / \mathrm{mL}$ ) using a densitometer (Grant Bio ${ }^{\text {TM }}$ DEN-1B, Grant Instruments Ltd.; Cambs SG8 $6 \mathrm{~GB}$, England). Bacterial counts were determined by spreading untreated controls of each experiment onto agar plates allowing exact calculations of surviving microorganisms as well as reduction rates.

\section{Test Wound Dressings and Preparation of Specimen}

Five commercial dressings were investigated, four antimicrobial dressings containing either octenidine di-hydochloride (OCT), polyhexanide (PHMB), cadexomer-iodine (C-IOD) or ionic silver (AG) and an agent-free control dressing. Detailed data on dressing composition, contained active agent and concentration are displayed in Table 1. For all dressings, round pieces with a diameter of $2.2 \mathrm{~cm}\left(\mathrm{~A}=3.8 \mathrm{~cm}^{2}\right)$ were prepared in an aseptic manner fitting press-fit in one well of a standard 12-well-plate 
TABLE 1 | Investigated dressings.

\begin{tabular}{|c|c|c|c|}
\hline & Manufacturer & Material & Active agent (concentration) \\
\hline UrgoClean $^{\oplus}$ & Urgo GmbH, Chenôve, France & $\begin{array}{l}\text { Cohesive polyabsorbent polyacrylate fibers; TLC wound } \\
\text { matrix }{ }^{\circledR} ; \text { non-adhesive }\end{array}$ & None \\
\hline Sorelex ${ }^{\circledR}$ & $\begin{array}{l}\text { Contipro C, Dolní Dobrouč, Czech } \\
\text { Republic }\end{array}$ & $\begin{array}{l}\text { Permeable, gel-forming bioactive gauze, releases octenidine } \\
\text { hydrochloride, and sodium hyluronat; non-adhesive }\end{array}$ & $\begin{array}{l}\text { Octenidine di-hydrochloride } \\
\text { (not indicated) }\end{array}$ \\
\hline $\begin{array}{l}\text { Suprasorb }{ }^{\circledR} \\
P+\mathrm{PHMB}\end{array}$ & $\begin{array}{l}\text { Lohmann\&Rauscher GmbH, } \\
\text { Rengsdorf, Germany; Vienna, Austria }\end{array}$ & $\begin{array}{l}\text { Semi permeable top film on polyurethane foam layer } \\
\text { impregnated with and releasing PHMB; non-adhesive }\end{array}$ & $\begin{array}{l}\text { Polyhexanide } \\
\left(0.25-0.65 \mathrm{mg} / \mathrm{cm}^{2}\right)\end{array}$ \\
\hline UrgoClean $^{\oplus} \mathrm{Ag}$ & Urgo GmbH, Chenôve, France & $\begin{array}{l}\text { Cohesive polyabsorbent polyacrylate fibers; TLC wound } \\
\text { matrix }{ }^{\circledR} \text {; silver ion coating; non-adhesive }\end{array}$ & Ionic silver $(0.50$ mg/cm²) \\
\hline $\begin{array}{l}\text { IODOSORB }^{\mathrm{TM}} \\
\text { Dressing }\end{array}$ & Smith \& Nephew GmbH, Hull, England & $\begin{array}{l}\text { Beats of cadexomer (dextrin and epichlorhydrin) on gauze } \\
\text { backing release iodine; non-adhesive }\end{array}$ & lodine $(0.90 \% \mathrm{w} / \mathrm{w})$ \\
\hline
\end{tabular}

Data of manufacturer, material, mechanism of action and contained substance concentration. Information has been obtained from the manufacturer.

(Sarstedt, Nuembrecht, Germany). For analyses, pieces of each dressing were placed in 12-well plates containing the lhBIOM and surrounding bacteria-rich serum, to create a wound-like scenario with direct contact to the biofilm.

\section{Preparation of Leucocyte Rich Human Plasma Biofilm Model (IhBIOM)}

The preparation of the IhBIOM was performed as described previously (Besser et al., 2020; Stuermer et al., 2021). In brief, fresh frozen plasma (FFP) of blood type $\mathrm{AB}$ (citrate buffered) and one LRS ${ }^{\circledR}$ chamber of leukocyte apheresis (Trima Accel ${ }^{\circledR}$; Terumo BCT Inc., Lakewood, United States) were obtained from the Institute for Transfusion Medicine (University Medical Center Hamburg-Eppendorf, Germany). Preparation of the lhBIOM was initiated on the same day of blood product donation. In brief, FFP was thawed, adjusted to $250 \mathrm{~mL}$ and the "immunocompetence" in form of leukocytes from the LRS ${ }^{\circledR}$ chamber was added. The immune cells were obtained by using a special automated blood collection system for apheresis (Terumo BCT design, Trima Accel $^{\circledR}$ LRS $^{\circledR}$ Platelet, Plasma Set, REF number 82300; Terumo BCT Inc., Lakewood, United States), which removes nearly all leukocytes of the donor from the platelet sample, so that its concentration is equivalent to about $40 \times 10^{3}$ leukocytes/ $\mu \mathrm{L}$. The content of one LRS $^{\circledR}$ chamber was placed in a tube, washed out with $3 \mathrm{~mL}$ of the FFP to remove any residual leukocytes, the wash-out added to the tube and centrifuged at 1,610 g. The layer of erythrocytes was gently removed and the remaining plasma-leukocyte mix added to the remaining FFP at room temperature. After gentle mixing, the bacterial suspension was added resulting in a final concentration of $1.5 \times 10^{6} \mathrm{cfu} /$ model. Next $18.26 \mu \mathrm{L} \mathrm{CaCl} 2(500 \mathrm{mM})$ per $\mathrm{mL}$ plasma was added to the bacteria-plasma-mix to induce coagulation. The resulting biofilm mixture was immediately transferred into 12 -well plates $(1.5 \mathrm{~mL}$ per model/well). Well plates were placed on a rotation shaker and incubated for $12 \mathrm{~h}$ at $60 \mathrm{rpm}$ and $37.0^{\circ} \mathrm{C}$ to polymerize and form an extracellular matrix. After this period, stable biofilm discs with integrated test organisms resulted. Procedures of blood product collection are in accordance with "good clinical practice" standards and all donors gave their informed and written consent for the use of their blood products.

\section{Dressing Performance on $P$. aeruginosa Biofilm and Quantification of Bacterial Load}

After $12 \mathrm{~h}$ of biofilm maturation in the lhBIOM (Figure 1), the test dressings were placed on the models as described above under "specimen preparation." Treatment with dressings was performed for 1,3 , or 6 days without dressing change. After the specified treatment periods, wound dressings were carefully removed so that neither residues of the dressing remained on the model, nor biofilm substance was lost. Plastic beakers $(50 \mathrm{~mL})$ were filled with glass beads $(\mathrm{D}=3-4 \mathrm{~mm})$, so that the bottom was slightly covered. $10 \mathrm{~mL}$ neutralizer solution TLSNt-SDS ( $6 \%$ polysorbate 80 , $6 \%$ saponin, $0.8 \%$ lecithin, $2 \%$ sodium dodecyl sulfat, $0.6 \%$ sodium thiosulfat in Aqua dest.) was added. The wound dressings were placed in the neutralizer solution. After shaking for $10 \mathrm{~min}$ at $200 \mathrm{rpm}$, extracts were plated out in 10 -fold dilutions on CSA and incubated at $37^{\circ} \mathrm{C}$ under aerobic conditions for $48 \mathrm{~h}$ before quantification of colony forming units using a digital colony counter (NSCA 436000, VWR International GmbH; Germany).

Antimicrobial activity in the wells was neutralized by adding $300 \mu \mathrm{L}$ of the neutralizing solution TLSNt-SDS to each well. Plates were subsequently placed on a rotation shaker for $5 \mathrm{~min}$ at room temperature for incubation of neutralizing agent. Subsequently, biofilm models were dissolved using bromelain (Bromelain from pineapple, Serva Electrophoresis $\mathrm{GmbH}$; Heidelberg, Germany). Bromelain solution was prepared using $2.1 \mathrm{~g}$ powder, dissolved in $100 \mathrm{~mL}$ phosphate buffered saline (PBS) and $1.5 \mathrm{~mL}$ was added to each well containing a biofilm model. The biofilms were punctured with sterile pipette tips several times to ensure and accelerate complete dissolution of the model. After $3 \mathrm{~h}$ incubation $\left(37^{\circ} \mathrm{C}\right.$; aerobic conditions) the biofilm models were completely dissolved with the exception of the iodine wound dressing models. These left insoluble residues. The microbial counts in the dissolved models were quantified in the same manner as the dressing eluates.

\section{Qualitative Evaluation by Scanning Electron Microscopy (SEM)}

To visualize the antimicrobial effects of the wound dressings on biofilm morphology and structure, scanning electron microscopy 


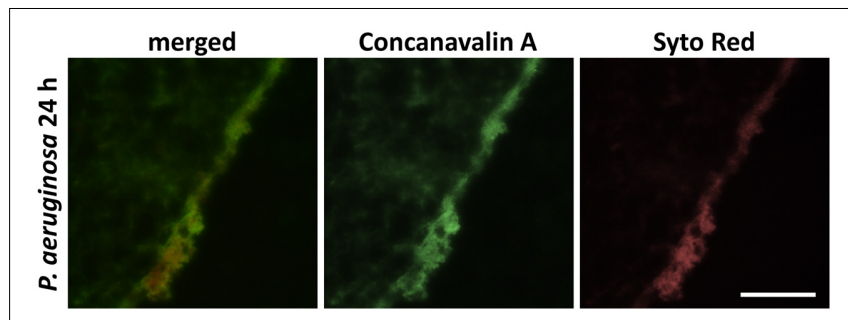

FIGURE 1 | Immunohistochemical carbohydrates-staining of the glycocalyx of $24 \mathrm{~h}$ maturated biofilm produced by $P$. aeruginosa. Carbohydrates were detected with FITC-conjugated Con A, cellular and bacterial nucleic acids with SYTO Red staining (scale bar: $100 \mu \mathrm{m}$ ).

(SEM) was performed. All dressings were carefully removed prior to further preparation of the models and antimicrobial activity were neutralized using the TLSNt-SDS neutralizing solution. Biofilm models were fixed with a glutaraldehyde/PVP-solution containing $2.5 \%$ glutaraldehyde, $2 \%$ polyvinylpyrrolidone (PVP) and $0.5 \% \mathrm{NaNO}_{2}$ in $0.1 \mathrm{M}$ cacodylate buffer for $1 \mathrm{~h}$ at $4^{\circ} \mathrm{C}$. After washing three times $(0.1 \mathrm{M}$ cacodylate buffer $)$ they were prepared with liquid nitrogen to get freeze fracture fragments and stored in $0.1 \mathrm{M}$ cacodylate buffer. In order to stain the glycoxalyx, the samples were incubated for $18 \mathrm{~h}$ at RT in an arginine- $\mathrm{HCl}$ solution (solution with $2 \%$ arginine- $\mathrm{HCL}$, glycine, sucrose and sodium glutamate). Next samples were rinsed again three times for $5 \mathrm{~min}$ with aqua dest. and subsequently stored for $5.5 \mathrm{~h}$ at RT in a mixture of $2 \%$ tannic acid and guanidine-HCL. After storage, samples were again rinsed once with aqua dest ( 5 min incubation) and three times with $0.1 \mathrm{M}$ cacodylate buffer (5 min incubation) and incubated overnight at $4^{\circ} \mathrm{C}$. After overnight incubation, samples were placed in a $1 \% \mathrm{OsO}_{4}$ solution for $30 \mathrm{~min}$ at $\mathrm{RT}$ followed by three rinsing steps with $0.1 \mathrm{M}$ cacodylate buffer (10 min incubation) and stored again over night at $4^{\circ} \mathrm{C}$. Last, samples were dehydrated in an isopropanol series (50, 70, 90, and 100\%) for $15 \mathrm{~min}$ each followed by an acetone series (50, 75, and 100\%) also each for $15 \mathrm{~min}$. The drying step was completed by a final treatment in the critical point dryer (BAL-TEC AG, Balzers, Liechtenstein). With a sputter coater (BAL-TEC AG, Balzers, Liechtenstein), samples were sputtered with gold palladium and afterward analyzed by Zeiss Sigma VP SEM (Zeiss, Oberkochen, Germany) at $2 \mathrm{kV}$ acceleration voltages using the in-lens and SE detectors.

\section{Statistical Analysis}

Data is expressed as means \pm standard error of the mean (SEM) based on triplicates derived from six different anonymous blood donors (FFP and leukocytes) at different time-points. Microbial reduction rates (in $\Delta \log _{10} \mathrm{cfu} / \mathrm{mL}$ ) were calculated and analyzed using the statistics program GraphPad PRISM (Version 9.0; GraphPad Software Inc., La Jolla, United States). For analysis of statistical significance a two-way repeated-measures ANOVA, followed by Tukey post hoc test for evaluation of multiple comparisons was applied. A $p$-value of $p \leq 0.05$ was considered statistically significant.

\section{RESULTS}

\section{Quantitative Microbial Load in Wound Dressing Eluate}

Over the examined treatment course of a total of 6 days, the microbial counts in the wound dressing eluate followed a directly proportional pattern to the microbial load in the solid biofilm itself. Under treatment with octenidine di-hydrochloride (OCT) and ionic silver (AG), a gradual increase of microbial counts on days 1, 3 and 6 were observed (Figure 2). Such increasing microbial counts also occurred in the control dressing (CTRL) with no antimicrobial agent, consequently showing no antibiofilm activity of OCT and AG on the wound dressing eluate. PHMB demonstrated a statistically non-significant decrease in microbial counts of the eluate on day $1(0.62 \pm 0.16$ $\log _{10}$ steps) compared to initial counts. Subsequently, the PHMB dressing maintained the level of microbial counts over the remaining course of 6 days compared to the increasing counts in the control dressing (Figure 2), demonstrating a bacteriostatic effect.

On day 1 , treatment with the $\mathrm{C}-\mathrm{IOD}$ dressing resulted in a statistically non-significant reduction of $1.51 \pm 0.41 \log _{10}$ steps compared to initial bacterial counts in the dressing eluate. After 3 and 6 days of treatment, C-IOD achieved a significant reduction of microbial counts in the eluate compared to initial counts of $2.93 \pm 0.29 \log _{10}$ steps $(p=0.0207)$ and $6.92 \pm 0.58 \log _{10}$ steps $(p=0.0154)$, respectively. Compared to the control dressing this accounts for a significantly reduced bacterial burden of $1.41 \pm 0.24 \log _{10}$ steps on day $1(p=0.0254), 3.51 \pm 0.42 \log _{10}$ steps on day $3(p=0.0069)$ and $7.62 \pm 0.60 \log _{10}$ steps on day 6 $(p=0.0077$; Figure 2).

\section{Anti-biofilm Activity of Antimicrobial Wound Dressings on the IhBIOM}

Regarding the antimicrobial effect on bacteria within the biofilm model, the results demonstrated an overall similar trend for wound dressings and dressing eluate (Figure 2). Treated with an OCT-containing dressing over a 6-day-period, microbial counts within the lhBIOM showed a continued increase with no observable antimicrobial effect. The AG-containing dressing demonstrated similar results, with the exception of an initial significant decrease in microbial counts on day 1 of $3.33 \pm 1.50$ $\log _{10}$ steps $(p=0.013)$ compared to initial counts. On day 3 however the initial reductive effect had worn off and microbial re-growth above initial counts had occurred.

After an initial non-significant reduction on day 1, the PHMBimpregnated dressing maintained a prolonged bacteriostatic effect. This was observed for the biofilm itself as well as in the dressing eluate. PHMB reduced the initial bacterial counts by $1.71 \pm 0.31 \log _{10}$ steps, subsequently keeping it at the reduced level over the course of 6 days without occurring re-growth (Figure 2). Compared to the control dressing, PHMB obtained a significant reduction in bioburden of $2.36 \pm 0.12 \log _{10}$ steps on day $3(p=0.0028)$ and $2.66 \pm 0.19 \log _{10}$ steps on day 6 $(p=0.0010)$ in the $1 \mathrm{hBIOM}$. The reduction on day 1 compared to the control dressing, however, was not statistically significant. 


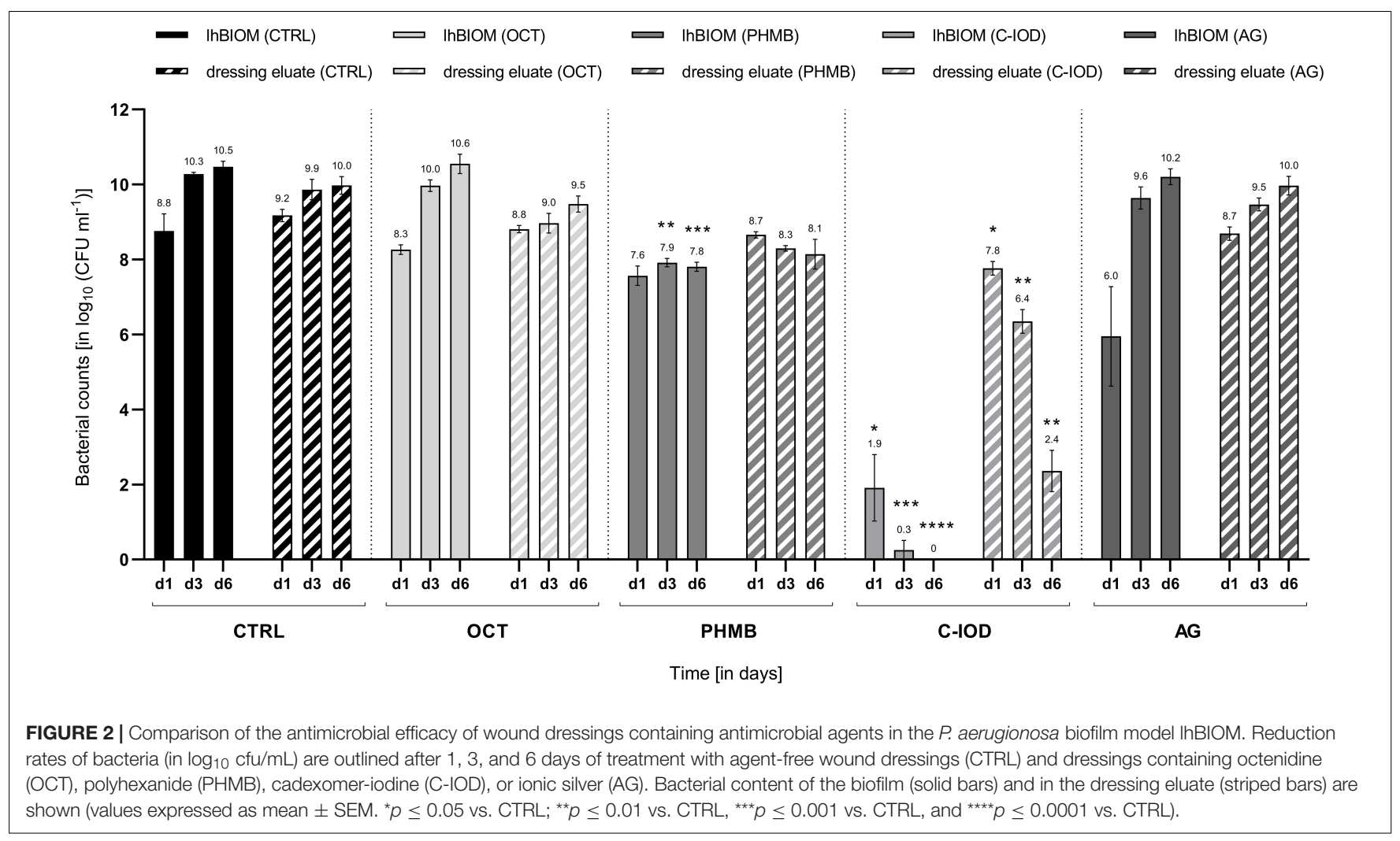

The C-IOD containing dressing showed the highest antibiofilm activity. On day 1 , bacterial counts in the biofilm were significantly reduced by $7.37 \pm 0.99 \log _{10}$ steps $(p=0.0387)$ compared to initial counts. On day 3 the initial bacterial load was reduced by $9.03 \pm 0.45 \log _{10}$ steps $(p=0.0054)$ and on day 6 no quantifiable microrganisms were retrieved, representing a complete reduction of initial microbial counts $\left(9.28 \pm 0.24 \log _{10}\right.$ steps; $p=0.0015$; Figure 2).

\section{Visual Analysis of Dressing Effects on the IhBIOM Using Scanning Electron Microscopy (SEM)}

SEM analysis of $P$. aeruginosa biofilm treated with wound dressings for 3 days (Figure 3 ) showed a distinct modification of the biofilm surface for all models compared to the untreated biofilm (Figure 3A). Whereas with drug-free wound dressings only an undulating fissuring with an almost intact surface was visible (Figure 3B), those containing antimicrobials induced an increased porosity. This was most prominent in the cadexomeriodine dressing (Figure 3E). The former induced a shotgunlike change in the biofilm surface reflecting the penetration of iodine from the cadexomer beads into deeper layers. The OCT- (Figure 3C) and PHMB-containing wound dressings (Figure 3D) showed a smooth surface with multiple isolated but rather big holes and their structural appearances were quite similar. The AG-containing dressing seemed to induce a more pronounced roughening of the biofilm surface with, however, fewer entry holes and an overall lower porosity (Figure 3F).

\section{DISCUSSION}

In guidelines, local antimicrobials such as different silver formulations, polyhexanide (PHMB), octenidine, dihydrochloride or iodine are rated as equally efficient (AWMF, 2012). Consensus about advantages and disadvantages in direct comparison, recommendations and independent analyses of efficacies in complex test scenarios or clinical conditions are, however, rare (Kramer et al., 2018). Even though several studies addressed this issue, most concluded that further analyses are mandatory to gain evidence-based recommendations for the daily use of antimicrobial dressings (Storm-Versloot et al., 2010; Daeschlein, 2013; Forster and Pagnamenta, 2015; Wu et al., 2015; Norman et al., 2016). Using a complex 3D model designed to mimic the micro-environment of a human wound biofilm (lhBIOM), composed of human material (plasma, platelets and leukocytes), this study aimed to extend the knowledge-base on efficacy profiles of antimicrobial wound dressings in a more transferable, "closer-to-reality" test scenario (Besser et al., 2020; Rembe et al., 2020; Stuermer et al., 2021). In this model, the incorporated bacteria encounter not only a milieu similar to the wound exudate (Loeffler et al., 2013), but also a certain immunological competence represented by leukocytes, so a biofilm matrix has to be produced under the challenge of a donor's immunocompetence (Figure 1).

The obtained results of the investigated wound dressings regarding antimicrobial efficacy partly contrast what is currently considered best-practice knowledge and has been previously reported. In case of the iodine-containing product (C-IOD), a 


\section{A}

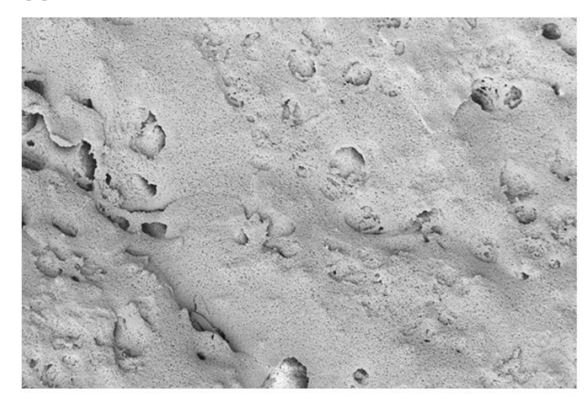

C

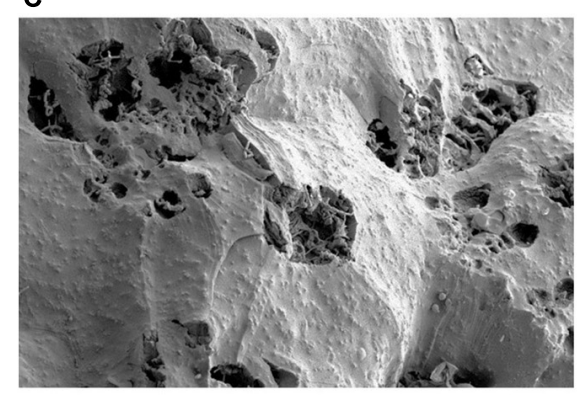

E

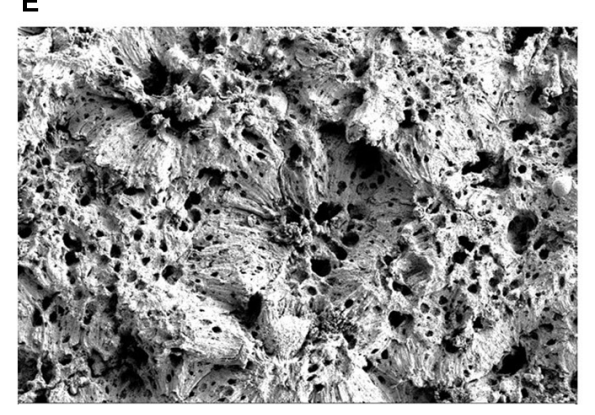

B

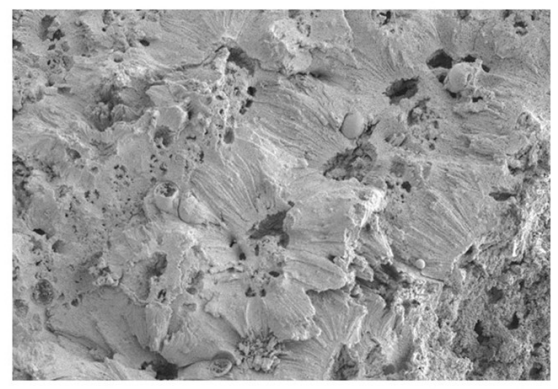

D

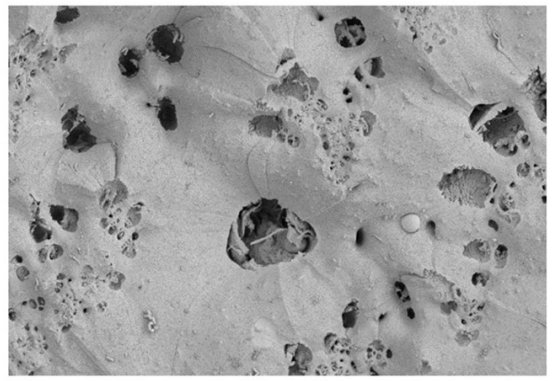

F

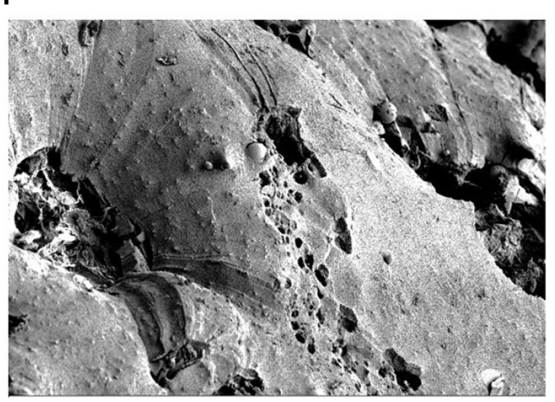

FIGURE 3 | Scanning electron microscopy (SEM) visualization of $P$. aerugionosa biofilm surface (IhBIOM) after 3 days under control conditions (A) and after treatment with commercial wound dressings: (B) polyurethane dressing with no active agent; (C) with octenidin (OCT); (D) with polyhexanide (PHMB); (E) with cadexomer-iodine (C-IOD) and (F) ionic silver. The untreated biofilm model (A) shows a densely connected surface structure. After treatment with cadexomer-iodine (E), surface structure appears rugged and "broken-open" with several holes as potential new entry points for iodine. After treatment with OCT (C) and PHMB (D) biofilm surface remains more dense though several holes are visible. Silver (F) induced the least changes.

distinct and continuous antimicrobial effect was observed in this study with a successive microbial reduction within 3 days (Figure 2), confirming previous in vitro and clinical studies (Phillips et al., 2015; Fitzgerald et al., 2017; Roche et al., 2019). It should be emphasized, however, that under treatment with the cadexomer-iodine dressing, the biofilm model was not completely dissolved in bromelain. This most likely arises from the proposed dehydrative effect caused by the cadoxmer agent (Akiyama et al., 2004; Fitzgerald et al., 2017). Judging by the reductive pattern in the solid biofilm model (lhBIOM), it seems, that the cadexomer-proportion of the dressing degrades and increases the porosity of the biofilm structure. Combined with iodine it acts partly lethal on exposed bacteria, while partly binding microorganisms within the dressing. This offers an explanation for the higher remaining microbial counts within the eluate on day 1 (Figure 2): Released microorganisms from the degraded biofilm structure are initially bound and subsequently reduced by the continued release of iodine. The simultaneous continued, slow release of iodine molecules into the degraded biofilm also reaches deeper structures and works against dorment bacteria. This approach is also supported by the SEM images, showing a failure of the closed protective EPS shield due to the impact of cadexomer-iodine.

Evaluations regarding the anti-biofilm activity of polyhexanide-containing wound dressings (PHMB) are rarely found in the literature (Huebner and Kramer, 2010; Huebner et al., 2010; Lenselink and Andriessen, 2011; Davis et al., 2017; Kramer et al., 2018); for the octenidine-containing wound dressing (OCT) no data at all could be found. Most efficacy assumptions and statements are transferred from analyses performed on microorganisms in a planktonic state or from evaluations of the antiseptic solution counterparts. Regarding 
the PHMB dressing, a reductive efficacy ( $2 \log _{10}$ steps) with a subsequent bacteriostatic effect could be demonstrated in this work, presumably due to the comparably high concentration of PHMB $\left(0.65 \mathrm{mg} / \mathrm{cm}^{2}\right)$ embedded in and released by the wound dressing (Figure 2 and Table 1). These results are in accordance with the results of earlier analyses of our working group, demonstrating a good efficacy for PHMB-containing antiseptics and antimicrobial irrigation solutions (Brackman and Coenye, 2016; Rembe et al., 2020). However, this positive quantitative effect is not reflected in the SEM images, since only slightly changes to the surface of the biofilm can be observed.

For the here tested silver-containing wound dressing (AG), the expected high antimicrobial efficacy could not be verified. While several in vitro evaluations in planktonic or simpler biofilm models demonstrated a good antimicrobial and anti-biofilm efficacy (O'Meara et al., 2000; Desroche et al., 2016; Percival et al., 2019b), these results could not be reproduced in a more complex in vitro biofilm scenario used here (Figure 2). Similar discrepancies and debates arise from various previous clinical studies of critically colonized or infected wounds, resulting in the necessity for further investigations (Lo et al., 2009; O'Meara et al., 2013; Dissemond et al., 2017). Even though a initial bacterial reduction was observed after 1 day of treatment, re-growth occurred over the following course of 6 days treatment, ultimately displaying similar microbial counts as the control dressing. Analyzing the eluate of the silver dressing, as a surrogate for the absorbed microorganism-containing wound exudate, there was also no reduction of the microbial load. These findings are contrary to previous descriptions by Desroche et al. (2016), stating that it "reduces the bacterial population and the biofilm of $P$. aeruginosa and MRSA up to $4 \log$ steps within 24 h for 7 days" (collagen I-coated surface with no human components). The AG-impregnated wound dressing contains ionic silver coated to the specific surface matrix in a comparably low concentration $\left(0.50 \mathrm{mg} / \mathrm{cm}^{2}\right.$; Table 1$)$. Besides the low concentration of the active agent, the composition of the milieu seemed to impede silver to exert its full antimicrobial effect. The relevance of its chemical structure has already been proven in previous studies. The loss of efficacy was pointed out for nanocrystalline silver (Gnanadhas et al., 2013; Rembe et al., 2018) more than for ionic silver, however, interactions with the wound microenvironment such as $\mathrm{pH}$ value or protein content have been described for both several times (Hirsch et al., 2011; Kapalschinski et al., 2013; Wiegand et al., 2015; Rembe et al., 2018). This emphasizes the need for human-adapted analyses in vitro, as the here tested dressing containing ionic silver demonstrates an overall loss of antimicrobial efficacy in a complex, protein-rich, human-adapted microenvironment.

Surprisingly, the octenidine-impregnated dressing showed no antimicrobial or anti-biofilm efficacy in the performed experiments, while the antiseptic solution tested in a similar complex model (hpBIOM) in previous studies, demonstrated the highest efficacy compared to other antimicrobial solutions (Besser et al., 2020; Rembe et al., 2020). In many previous studies, both against planktonic bacteria as well as biofilm, the active agent of the disinfectant Octenisept $t^{\circledR}$ has repeatedly proven to be highly effective (Brackman and Coenye, 2016;
Rembe et al., 2020), even though the onset of its full efficacy was shown to be delayed in complex biofilm scenarios (up to $48 \mathrm{~h}$ ). The missing antimicrobial activity of the OCT dressing might therefore be attributed to the dressing configuration with a retained release of the active agent into the wound (here biofilm model) or an insufficient amount of active agent embedded and released to achieve an impact. Unfortunately, the exact amount of octenidine di-hydrochloride in the dressing is not provided in the literature or by the manufacturer. However, the question of substance concentration has shown differences in antimicrobial dressing performance in previous studies, with lower concentrations yielding lower reduction rates (Rembe et al., 2018). Another restrictive factor of the dressing configuration might be the combination of octenidine, dihydrochloride and hyaluronic acid in the wound contact layer, which upon contact with wound exudate forms a gelling structure. This can lead to an "entrapment" of the active agent OCT in the gelling layer with the main antimicrobial activity exerted on microorganisms absorbed with the wound exudate into this layer and therefore low release of the agent into the wound and biofilm. Additionally, in the here tested dressing, only octenidine di-hydrochloride is embedded, in contrast to the highly effective liquid antiseptic Octenisept ${ }^{\circledR}$, which contains a combination of octenidine di-hydrochloride and phenoxyethanol. The additive phenoxyethanol, however, exerts additional antimicrobial effects and further contained additives as well as alcohols have been specifically attributed with biofilm degrading properties (Percival et al., 2017a,b, 2019a), therefore offering another explanatory factor for the missing antimicrobial and anti-biofilm efficacy in the presented results. The question whether a daily or 2-day dressing change, would enhance the performance of the less effective test dressings (AG and OCT) cannot be conclusively answered herein. In relation, however, the more effective dressings (PHMB and C-IOD) would expectedly also profit from a more frequent change regimen, therefore not altering the comparative performance.

The aspects regarding active substance concentration and physicochemical release patterns as well as specific interactions due to dressing and microenvironment composition are transferable and applicable to all here tested wound dressings. Supposedly, the specific constellation of such factors for individual dressings dictate the overall antimicrobial and anti-biofilm efficacy observed in the presented results. Here, the polyhexanide-containing (PHMB) and especially the cadexomer-iodine containing dressing (C-IOD) proved to be most active against $P$. aeruginosa biofilm compared to other antimicrobial wound dressing formulations. However, only cadexomer-iodine achieved an actual relevant bactericidal effect. This is also reflected in the SEM showing an increased porosity and a distinct alteration of the biofilm surface pattern. Similar results were observed in earlier research approaches regarding its anti-biofilm activity (Phillips et al., 2015). As mentioned earlier, the combined cadexomer-iodine dressing exerts its effect by two main aspects: Cadexomer directly destroys biofilm structures by collapsing the bacterial glycocalyx (EPS) which is composed of 99\% water (Lapping-Scott et al., 2014) by dehydration through water absorbtion, while iodine as an antimicrobial agent can subsequently eliminate bacteria (here 
P. aeruginosa) released and exposed from the damaged biofilm structure (Akiyama et al., 2004).

Naturally, the presented results are limited as to the fact that they are in vitro results, calling for a careful consideration in terms of translation to clinical situations. Due to the experimental setup using a complex, human material based approach, the evaluations have been moved one step closer to clinical reality, however, still need to be interpreted with caution and in relation. The here used model allows for far more precise interpretations of results in terms of transferability into reality than simpler models. Still, even more complex test settings (incorporating human cell lines and three-dimensional tissue structures) will be required in the future to even better interpret complex efficacy interactions of antimicrobial products. The same goes for welldesigned, sufficiently powered randomized controlled clinical trial to back in vitro findings and establish long-sought evidencebased clinical guidelines. The present results, however, show that these are urgently needed to clarify indications and support correct choice of wound care products.

Further limitations include the intentional disregard of the negative aspect of cytotoxicity potentially caused to the regenerating wound by excessive release of antimicrobial substances from wound dressings, as well as the evaluation of only one dressing per agent group with only a single combination of substance concentration and dressing formation per group investigated. Cytotoxic aspects were intentionally not addressed in this work due to focus being placed on bactericidal effects against biofilms, which untreated would exert potentially more harm to the healing wound than a confined cytotoxic impact derived from an antimicrobial treatment.

Finally, questions regarding the relevance of physicochemical release patterns and the specific composition and placement of the active agent within the dressing would be better addressed comparing differently manufactured dressings. However, in most cases (OCT, C-IOD, and PHMB) only very limited or even no further dressings containing the specific antimicrobial agents are commercially available and therefore need to be specifically manufactured as prototypes. This represents a continuous future endeavor in the field of material science, to identify and reliably validate the best combination of wound dressing material and active antimicrobial agent.

\section{CONCLUSION}

In this in vitro study the challenge a wound biofilm poses for antimicrobial agents becomes evident once again. The iodine-

\section{REFERENCES}

Akiyama, H., Oono, T., Saito, M., and Iwatsuki, K. (2004). Assessment of cadexomer iodine against Staphylococcus aureus biofilm in vivo and in vitro using confocal laser scanning microscopy. J. Dermatol. 31, 529-534. doi: 10. 1111/j.1346-8138.2004.tb00549.x

AWMF. (2012). Local therapy of chronic wounds in patients with the risk of peripheral arterial disease, diabetes or chronic venous insufficiency. Reg.No. 091-001. Available Online at: www.awmf.org/leitlinien/detail/11/091-001. html and polyhexanide-containing dressings perform as expected with a high bactericidal effect of C-IOD and a sustained bacteriostatic effect of PHMB over the course of 6 days even though not all surviving bacteria were counted due to incomplete lysis of the biofilm under C-IOD. The silver- and octenidine-containing wound dressings on the contrary did not show a bactericidal or bacteriostatic activity in the employed complex biofilm model (lhBIOM). In all wound dressings, the overall composition of the dressing, the concentration of the active substance and the form of interaction with the microenvironment are postulated to be crucial factors. For future anti-biofilm treatment strategies, especially dressings should be sought out, that exhibit biofilm/EPS degrading as well as antimicrobial properties, either in a single active substance or combinations. Therefore, biocompatible active agents or additives, that are readily and continuously released into the wound to interact with the biofilm need to be further investigated.

\section{DATA AVAILABILITY STATEMENT}

The raw data supporting the conclusions of this article will be made available by the authors, without undue reservation.

\section{AUTHOR CONTRIBUTIONS}

ES, FB, and RS designed the study. IP, VW, AU, MD, and MG carried out the experiments. ES, J-DR, AK, and RS analyzed the results and performed statistical analysis. IP, MD, and EN made the REM pictures and figures. ES, J-DR, SD, and RS drafted the manuscript. All authors contributed to the article and approved the submitted version.

\section{FUNDING}

The presented work was funded by the German Initiative Chronische Wunden e.V. (ICW).

\section{ACKNOWLEDGMENTS}

We would like to thank Mrs. A. Borger for her support in generating the blood agents, and $\mathrm{B}$. Haußmann for preparation of the biofilm models for SEM analyzes.

Besser, M., Dietrich, M., Weber, L., Rembe, J. D., and Stuermer, E. K. (2020). Efficiency of antiseptics in a novel 3-dimensional human plasma biofilm model (hpBIOM). Sci. Rep. Nat. Res. 10:4792. doi: 10.1038/s41598-02061728-2

Bowler, P. G., Duerden, B. I., and Armstrong, D. G. (2001). Wound microbiology and associated approaches to wound management. Clin. Microbiol. Rev. 14, 244-269.

Brackman, G., and Coenye, T. (2016). In vitro and in vivo biofilm wound models and their application. Adv. Exp. Med. Biol. 897, $15-32$. 
Daeschlein, G. (2013). Antimicrobial and antiseptic strategies in wound management. Int. Wound J. 10, 9-14.

Davis, S. C., Harding, A., Gil, J., Parajon, F., Valdes, J., Solis, M., et al. (2017). Effectiveness of a polyhexanide irrigation solution on methicillin-resistant Staphylococcus aureus biofilms in a porcine wound model. Int. Wound J. 14, 937-944. doi: 10.1111/iwj.12734

Demidova-Rice, T. N., Hamblin, M. R., and Herman, I. M. (2012). Acute and impaired wound healing: pathophysiology and current methods for drug delivery, part 1: normal and chronic wounds: biology, causes, and approaches to care. Adv. Skin Wound Care 25, 304-314. doi: 10.1097/01.ASW.0000416006. 55218.d0

Desroche, N., Dropet, C., Janod, P., and Guzzo, J. (2016). Antibacterial properties and reduction of MRSA biofilm with a dressing combining polyabsorbent fibres and a silver matrix. J. Wound Care. 25, 577-584. doi: 10.12968/jowc.2016.25. 10.577

Dissemond, J., Boettrich, J. G., Braunwarth, H., Hilt, J., Wilken, P., and Münter, K. C. (2017). Evidence for silver in wound care - meta-analysis of clinical studies from 2000-2015. J. Dtsch. Dermatol. Ges. 15, 524-535. doi: 10.1111/ddg. 13233

Fitzgerald, D. J., Renick, P. J., Forrest, E. C., Tetens, S. P., Earnest, D. N., and McMillan, J. (2017). Cadexomer iodine provides superior efficacy against bacterial wound biofilms in vitro and in vivo. Wound Repair. Regen. 25, 13-24. doi: $10.1111 /$ wrr. 12497

Forster, R., and Pagnamenta, F. (2015). Dressings and topical agents for arterial leg ulcers. Cochrane Database Syst. Rev. 6:CD001836.

Gnanadhas, D. P., Thomas, M., Thomas, R., Raichur, A. M., and Chakravortty, D. (2013). Interaction of silver nanoparticles with serum proteins affects their antimicrobial activity in vivo. Antimicrob. Agent. Chemother. 57, 4945-4955. doi: 10.1128/AAC.00152-13

Guest, J. F., Ayoub, N., McIlwraith, T., Uchegbu, I., Gerrish, A., Weidlich, D., et al. (2017). Health economic burden that different wound types impose on the UK's national health service. Int. Wound J. 14, 322-330. doi: 10.1111/iwj. 12603

Guest, J. F., Fuller, G. W., and Vowden, P. (2018). Diabetic foot ulcer management in clinical practice in the UK: costs and outcomes. Int. Wound J. 5, 43-52.

Hirsch, T., Limoochi-Deli, S., Lahmer, A., Jacobsen, F., Goertz, O., Steinau, H. U., et al. (2011). Antimicrobial activity of clinically used antiseptics and wound irrigating agents in combination with wound dressings. Plast. Reconstr. Surg. 127, 1539-1545. doi: 10.1097/PRS.0b013e318208d00f

Huebner, N. O., and Kramer, A. (2010). Review on the efficacy, safety and clinical applications of polihexanide, a modern wound antiseptic. Skin Pharmacol. Physiol. 23, 17-27. doi: 10.1159/000318264

Huebner, N. O., Matthes, R., Koban, I., Raendler, C., Mueller, G., Bender, C., et al. (2010). Efficacy of chlorhexidine, polihexanide and tissue-tolerable plasma against Pseudomonas aeruginosa biofilms grown on polystyrene and silicone materials. Skin. Pharmacol. Physiol. 23, 28-34. doi: 10.1159/000 318265

James, G. A., Swogger, E., Wolcott, R., Pulcini, E., Secor, P., Sestrich, J., et al. (2008). Biofilms in chronic wounds. Wound Repair. Regen. 16, 37-44.

Kapalschinski, N., Seipp, H. M., Onderdonk, A. B., Goertz, O., Daigeler, A., Lahmer, A., et al. (2013). Albumin reduces the antibacterial activity of polyhexanide-biguanide-based antiseptics against Staphylococcus aureus and MRSA. Burns 39, 1221-1225. doi: 10.1016/j.burns.2013.03.003

Kirketerp-Møller, K., Stewart, P. S., and Bjarnsholt, T. (2020). The zone model: a conceptual model for understanding the microenvironment of chronic wound infection. Wound Repair. Regen. 28, 593-599. doi: 10.1111/wrr. 12841

Koburger, T., Hubner, N. O., Braun, M., Siebert, J., and Kramer, A. (2010). Standardized comparison of antiseptic efficacy of triclosan, PVP-iodine, octenidine dihydrochloride, polyhexanide and chlorhexidine digluconate. J. Antimicrob. Chemother. 65, 1712-1719.

Kramer, A., Dissemond, J., Kim, S., Willy, C., Mayer, D., Papke, R., et al. (2018). Consensus on wound antisepsis: update 2018 skin pharmacol. Physiology 31, 28-58. doi: 10.1159/000481545

Lapping-Scott, H., Burton, S., and Stoodley, P. (2014). Revealing a world of biofilms-the pioneering research of bill costerton. Nat. Rev. Microbiol. 12, 781-787. doi: $10.1038 /$ nrmicro3343
Lenselink, E., and Andriessen, A. (2011). A cohort study on the efficacy of a polyhexanide-containing biocellulose dressing in the treatment of biofilms in wounds. J. Wound Care. 20, 534-536. doi: 10.12968/jowc.2011.20. 11.534

Lo, S. F., Chang, C. J., Hu, W. Y., Hayter, M., and Chang, Y. T. (2009). The effectiveness of silver-releasing dressings in the management of non-healing chronic wounds: a meta-analysis. J. Clin. Nurs. 18, 716-728. doi: 10.1111/j. 1365-2702.2008.02534.x

Loeffler, M. W., Schuster, H., Bühler, S., and Beckert, S. (2013). Wound fluid in diabetic foot ulceration: more than just an undefined soup? Int. J. Low Extrem. Wounds. 12, 113-129. doi: 10.1177/1534734613489989

Magill, S. S., Edwards, J. R., Bamberg, W., Beldavs, Z. G., Dumyati, G., Kainer, M. A., et al. (2014). Multistate point-prevalence survey of health care-associated infections. N. Engl. J. Med. 370, 1198-1208.

Norman, G., Dumville, J. C., Moore, Z. E., Tanner, J., Christie, J., and Goto, S. (2016). Antibiotics and antiseptics for pressure ulcers. Cochrane Database Syst. Rev. 4, CD011586.

O’Meara, S., Al-Kurdi, D., Ologun, Y., Ovington, L. G., Martyn-St James, M., and Richardson, R. (2013). Antibiotics and antiseptics for venous leg ulcers. Cochrane Database Syst. Rev. 23:CD003557. doi: 10.1002/14651858.CD0 03557

O'Meara, S., Cullum, N., Majid, M., and Sheldon, T. (2000). Systematic reviews of wound care management: (3) antimicrobial agents for chronic wounds; (4) diabetic foot ulceration. Health Technol. Assess. 4, 1-237.

Percival, S. L., Mayer, D., Kirsner, R. S., Schultz, G., Weir, D., Roy, S., et al. (2019a). Surfactants: role in biofilm management and cellular behaviour. Int. Wound J. 16, 753-760. doi: 10.1111/iwj.13093

Percival, S. L., Mayer, D., Malone, M., Swanson, T., Gibson, D., and Schultz, G. (2017a). Surfactants and their role in wound cleansing and biofilm management. J. Wound Care. 26, 680-690. doi: 10.12968/jowc.2017.26.11.680

Percival, S. L., Mayer, D., and Salisbury, A. M. (2017b). Efficacy of a surfactantbased wound dressing on biofilm control. Wound Repair. Regen. 25, 767-773. doi: 10.1111/wrr.12581

Percival, S. L., McCarty, S. M., and Lipsky, B. (2015). Biofilms and wounds: an overview of the evidence. Adv. Wound Care 4, 373-381. doi: 10.1089/wound. 2014.0557

Percival, S. L., Salisbury, A. M., and Chen, R. (2019b). Silver, biofilms and wounds: resistance revisited. Crit. Rev. Microbiol. 45, 223-237. doi: 10.1080/1040841X. 2019.1573803

Phillips, P. L., Yang, Q., Davis, S., Sampson, E. M., Azeke, J. I., Hamad, A., et al. (2015). Antimicrobial dressing efficacy against mature Pseudomonas aeruginosa biofilm on porcine skin explants. Int. Wound J. 12, 469-483. doi: 10.1111/iwj. 12142

Rembe, J. D., Fromm-Dornieden, C., Böhm, J. K., and Stuermer, E. K. (2018). The influence of human acute wound fluid (AWF) on the antibacterial efficacy of different antiseptic polyurethane foam dressings: an in-vitro analysis. Wound Repair. Regen. 26, 27-35. doi: 10.1111/wrr.12612

Rembe, J. D., Huelsboemer, L., Besser, M., and Stuermer, E. K. (2020). Anti-biofilm activity of antimicrobial hypochlorous wound irrigation solutions compared to common wound antiseptics and bacterial resilience in a novel in-vitro human plasma biofilm model (hpBIOM). Front. Microbiol. 11:564513. doi: 10.3389/ fmicb.2020.564513

Roche, E. D., Woodmansey, E. J., Yang, Q., Gibson, D. J., Zhang, H., and Schultz, G. S. (2019). Cadexomer iodine effectively reduces bacterial biofilm in porcine wounds ex vivo and in vivo. Int. Wound J. 16, 674-683.

Shukla, S. K., Sharma, A. K., Gupta, V., Kalonia, A., and Shaw, P. (2020). Challenges with wound infection models in drug development. Curr. Drug Targets 21, 1301-1312. doi: 10.2174/1389450121666200302093312

Sibbald, R. G., Coutts, P., and Woo, K. Y. (2011). Reduction of bacterial burden and pain in chronic wounds using a new polyhexamethylene biguanide antimicrobial foam dressing-clinical trial results. Adv. Skin. Wound Care. 24, 78-84. doi: 10.1097/01.ASW.0000394027.82702.16

Storm-Versloot, M. N., Vos, C. G., Ubbink, D. T., and Vermeulen, H. (2010). Topical silver for preventing wound infection. Cochrane Database Syst. Rev. 3:CD006478.

Stuermer, E. K., Besser, M., Brill, F. H. H., Geffken, M., Plattfaut, I., Severing, A. L., et al. (2021). Comparative analysis of biofilm models to determine the efficacy of antimicrobials. J. Hyg. Environ. Health. 234:113744. 
To, E., Dyck, R., Gerber, S., Kadavil, S., and Woo, K. Y. (2016). The effectiveness of topical polyhexamethylene biguanide (PHMB) agents for the treatment of chronic wounds: a systematic review. Surg. Technol. Int. 29, 45-51.

Wiegand, C., Abel, M., Ruth, P., Elsner, P., and Hipler, U. C. (2015). pH influence on antibacterial efficacy of common antiseptic substances. Skin Pharmacol. Physiol. 28, 147-158. doi: 10.1159/000367632

Wu, L., Norman, G., Dumville, J. C., O’Meara, S., and Bell-Syer, S. E. (2015). Dressings for treating foot ulcers in people with diabetes: an overview of systematic reviews. Cochrane Database Syst. Rev. 7: CD010471.
Conflict of Interest: The authors declare that the research was conducted in the absence of any commercial or financial relationships that could be construed as a potential conflict of interest.

Copyright (c) 2021 Stuermer, Plattfaut, Dietrich, Brill, Kampe, Wiencke, Ulatowski, Geffken, Rembe, Naumova, Debus and Smeets. This is an open-access article distributed under the terms of the Creative Commons Attribution License (CC BY).

The use, distribution or reproduction in other forums is permitted, provided the original author(s) and the copyright owner(s) are credited and that the original publication in this journal is cited, in accordance with accepted academic practice. No use, distribution or reproduction is permitted which does not comply with these terms. 